\title{
Realização de oficinas acadêmicas pelo grupo PET Odontologia: Um relato de experiência
}

\author{
Academic workshops held by the PET Dentistry group: An experience report \\ Talleres académicos realizados por el grupo PET Odontologia: Un relato de experiencia
}

Maria Eduarda Fernandes

ORCID: https://orcid.org/0000-0003-0867-5232 Universidade Estadual de Maringá, Brasil

E-mail: mariaeduardafernaandes@gmail.com

Eduarda Letícia Pagliosa

ORCID: https://orcid.org/0000-0002-3160-7062 Universidade Estadual de Maringá, Brasil

E-mail: pagliosaeduarda@gmail.com

Luisa Gonçalves Cardoso

ORCID: https://orcid.org/0000-0002-2182-707X Universidade Estadual de Maringá, Brasil

E-mail: luisagcardoso1@gmail.com

Maicom Colombo Júnior

ORCID: https://orcid.org/0000-0001-8014-6445

Universidade Estadual de Maringá, Brasil

E-mail: maicomjr98@gmail.com

Gabriela Steckel Neme

ORCID: https://orcid.org/0000-0003-1986-0206 Universidade Estadual de Maringá, Brasil

E-mail: gabrielasteckelneme@gmail.com

Laura Moretto Molina

ORCID: https://orcid.org/0000-0001-9815-7775 Universidade Estadual de Maringá, Brasil

E-mail: laurammolina1@gmail.com

Camila Fernanda Vasconcelos

ORCID: https://orcid.org/0000-0002-1597-9448

Universidade Estadual de Maringá, Brasil

E-mail: camilafv12@gmail.com

Flávia Akemi Nakayama Henschel

ORCID: https://orcid.org/0000-0003-3186-3346

Universidade Estadual de Maringá, Brasil

E-mail: flaviaakemi95@gmail.com

Weslley Souza Petyk

ORCID: https://orcid.org/0000-0002-4821-2200

Universidade Estadual de Maringá, Brasil

E-mail: weslleypetyk@gmail.com

Daniela Suemi Kamikawa

ORCID: https://orcid.org/0000-0002-7152-0500

Universidade Estadual de Maringá, Brasil

E-mail: danielasuemi97@gmail.com

Carla Militão Ricken

ORCID: https://orcid.org/0000-0002-9174-590X

Universidade Estadual de Maringá, Brasil

E-mail: carlaricken7@gmail.com

Renata Yumi Takahashi

ORCID: https://orcid.org/0000-0003-2845-4824

Universidade Estadual de Maringá, Brasil

E-mail: reyumi.takahashi@gmail.com

Leonardo Galvão da Silva Garcia

ORCID: https://orcid.org/0000-0002-5717-8040

Universidade Estadual de Maringá, Brasil

E-mail: ra109875@uem.br

Mariana Podadeiro de Andrade

ORCID: https://orcid.org/0000-0001-7903-2168

Universidade Estadual de Maringá, Brasil

E-mail: marianapodadeiro@gmail.com 


\title{
Resumo
}

O Programa de Educação Tutorial (PET) do curso de Odontologia da Universidade Estadual de Maringá (UEM), elabora oficinas acadêmicas com conteúdos multidisciplinares, buscando enriquecer a formação da graduação. Assim, proporciona uma forma de ensino, que envolve todos os participantes em tarefas e atividades, favorecendo o espaço de discussão e trocas de saberes. Portanto, a finalidade do presente trabalho é relatar a experiência da oficina acadêmica do grupo PET Odontologia UEM. As oficinas são organizadas pelos próprios petianos, nas quais são realizadas palestras/workshops sobre alguns temas previamente decididos em reunião administrativa com os integrantes e o tutor do grupo. Foram realizadas quatro edições, e em cada uma delas, foram abordados dois temas, separando um dia para cada temática. Dentre elas estão: a formatação de painéis, PowerPoint, resumos, oratória, confecção e desenvolvimento de planilhas, como administrar melhor o tempo, regras básicas da câmera fotográfica para o clínico e noções básicas de photoshop, comunicação básica em libras e organização do currículo lattes. As oficinas foram ministradas aos graduandos previamente inscritos, que levaram os materiais necessários para a realização das tarefas. Ao final dos eventos, foram distribuídas avaliações a serem preenchidas pelos participantes, proporcionando um positivo feedback ao grupo. Tendo em vista os diversos benefícios que a oficina acadêmica traz para a graduação, como o estímulo ao conhecimento, é de suma importância o trabalho do grupo PET na capacitação de uma ampla formação acadêmica.

Palavras-chave: Metodologia; Odontologia; Materiais de ensino.

\begin{abstract}
The Tutorial Education Program (PET) of the Dentistry course at the State University of Maringá (UEM), prepares academic workshops with multidisciplinary content, seeking to enrich the undergraduate education. Thus, it provides a form of teaching, which involves all participants in tasks and activities, favoring the space for discussion and exchange of knowledge. Therefore, the aim of the present paper is to report the experience of the academic workshop of the PET Dentistry UEM group. The workshops are organized by the members of the PET group themselves, in which lectures / workshops are held on some topics previously decided in an administrative meeting with the members and tutor of the group. Four editions have already been held and in each of them two themes were addressed, setting aside a day for each theme. Among them are: formatting panels, PowerPoint, summaries, oratory, making and developing spreadsheets, how to better manage time, basic rules of the camera for the clinician and basics of photoshop, basic communication in pounds and organization of the lattes curriculum. Workshops were given to previous registered undergraduates that took the necessary materials to do the tasks. At the end of the events, evaluations were distributed to be completed by the participants, providing positive feedback to the group. In view of the various benefits that the academic workshop brings to undergraduate courses, such as stimulating knowledge, the work of the PET group in training a broad academic background is of paramount importance.
\end{abstract}

Keywords: Methodology; Dentistry; Teaching materials.

\section{Resumen}

El Programa de Educación Tutorial (PET) del curso de Odontología de la Universidad Estatal de Maringá (UEM), prepara talleres académicos con temas multidisciplinarios, buscando enriquecer la formación de pregrado. De esta forma, proporciona una forma de enseñanza, que involucra a todos los participantes en tareas y actividades, favoreciendo el espacio de discusión e intercambio de conocimientos. Por tanto, el propósito del presente trabajo es dar a conocer la experiencia del taller académico del grupo PET Odontología UEM. Los talleres son organizados por los propios miembros del grupo, en los que se realizan charlas / talleres sobre algunos temas previamente decididos en una reunión administrativa con los alumnos y tutor del grupo. Ya se han realizado cuatro ediciones y en cada una de ellas se abordaron dos contenidos, reservando un día para cada tema. Entre ellos se encuentran: formateo de paneles, PowerPoint, resúmenes, oratoria, elaboración y desarrollo de hojas de cálculo, cómo administrar mejor el tiempo, reglas básicas de la cámara para el clínico y conceptos básicos de photoshop, comunicación básica en libras y 
organización del currículum de lattes. Los talleres fueron realizados a estudiantes de pregrado previamente matriculados, quienes llevaron los materiales necesarios para realizar las tareas. Al finalizar los eventos, se distribuyeron evaluaciones para ser completadas por los participantes, brindando retroalimentación positiva al grupo. En vista de los diversos beneficios que el taller académico aporta a los cursos de pregrado, como la estimulación del conocimiento, la labor del grupo PET en la formación de una formación académica ampliada es de suma importancia.

Palabras clave: Metodología; Odontología; Materiales de enseñanza.

\section{Introdução}

A promoção de oficinas acadêmicas realizadas pelo Programa de Educação Tutorial (PET) busca, conforme explicitado na Portaria $n^{\circ}$ 976-2010-MEC e ratificado na Portaria $n^{\circ}$ 343-2013-MEC, formular novas estratégias de desenvolvimento e modernização do ensino superior no país, além de estimular o espírito crítico de seus participantes. As vivências metodológicas proporcionadas pelo grupo no ensino, na pesquisa e na extensão, aliadas à perspectiva da pedagogia libertadora, apontam que a participação no programa tenha sido um diferencial benéfico na formação de seus egressos (Morel et al., 2020).

O grupo PET Odontologia da Universidade Estadual de Maringá (UEM) que foi instituído em 1994 é composto por 18 integrantes (petianos), destes 17 graduandos e um professor tutor, que têm como propósito fornecer uma melhor formação profissional e cidadã a graduação (Manholer et al., 2021). As diretrizes do PET, se implementadas corretamente, complementam as perspectivas tradicionais de educação e ajudam os alunos a se tornarem mais independentes no gerenciamento de suas necessidades de aprendizagem (Drebes et al., 2016 como citado em Dantas, 1995). Assim, os discentes podem desenvolver habilidades como autonomia, proatividade e cooperativismo quando são formados de maneira ativa, como agentes do seu próprio aprendizado (Nascimento \& Feitosa, 2020).

Uma oficina pode ser considerada uma estratégia de ensino que consiste em uma atividade prática e que deve ser realizada em grupo, contendo um momento de reflexão, em que o estudante se torna um sujeito ativo na construção de seu próprio conhecimento (Marcondes, 2008; Sousa, 2016). É, portanto, uma metodologia que combina teoria e prática, trazendo consigo uma proposta de ensino inovadora e eficaz ao confrontar os participantes com o objeto de estudo em questão, instigando o aprendizado (Joaquim et al., 2020). Ademais, proporciona o envolvimento de todos os participantes em tarefas e atividades que favorecem espaços de discussão, trocas de experiências pessoais e do grupo a partir de uma horizontalidade na formação do saber, baseada no tripé: sentir-pensar-agir (Paviani, 2009). Desse modo, dinâmicas como oficinas permitem ao aluno vivenciar experiências que não estão presentes nos modelos curriculares convencionais, o que enriquece a formação global, propiciando condições melhores para o mercado profissional ou pós-graduação (Meneghel et al., 2017).

Existem várias maneiras diferentes de ministrar uma oficina, entretanto mesmo seguindo o planejamento inicial, há possibilidade de que novos desdobramentos transcorram. Por isso, é preciso estar atento a realização da tarefa e ao mesmo tempo, aberto a mudanças (Pey, 1997). Neste contexto, a finalidade do presente trabalho foi relatar a experiência do grupo PET Odontologia UEM no desenvolvimento e na aplicação da atividade intitulada "Oficina Acadêmica do grupo PET Odontologia UEM".

\section{Metodologia}

Frente à dificuldade geral dos graduandos em confecção de resumos, painel, apresentação em Power Point, organização do currículo lattes, falar em público, elaborar planilhas no Excel, editar fotos, utilizar câmera fotográfica e se comunicar com pacientes que possuem limitações auditivas, o grupo PET Odontologia UEM buscou, com a elaboração de oficinas acadêmicas, auxiliar os alunos nesses temas, e explanar as dúvidas frequentes.

Para organizar a atividade, os temas foram previamente decididos em reunião administrativa com os petianos. O 
grupo foi subdividido a fim de que cada integrante ficasse responsável por uma função específica na organização. As oficinas dividiam-se em dois dias seguidos, com temas distintos em cada um, com duração máxima de 2 horas. A divulgação da oficina para a graduação deu-se por meio de cartazes e postagens em redes sociais.

A oficina iniciava com uma breve explanação feita por alguns petianos designados a ministrar a atividade, contando ou não com a ajuda de um especialista no assunto. Na sequência, os alunos realizaram em seus próprios notebooks e/ou materiais disponibilizados algumas ações da aula expositiva. As dúvidas foram esclarecidas pelos petianos monitores que previamente calibrados e capacitados, ajudaram os participantes da oficina.

Nas três primeiras edições da oficina que ocorreram presencialmente em 2017, 2018 e 2019, as inscrições foram feitas das 17 às 18 horas, por ordem de chegada, e início das atividades às 18 horas, sendo que a inscrição de um dia não era válida para o dia seguinte. As palestras, com temáticas: Confecção de resumo científico, painéis e apresentações no PowerPoint, Currículo Lattes, Oratória, Excel, Aprenda a administrar seu Tempo e Regras básicas da câmera fotográfica para o clínico e noções básicas de photoshop, foram realizadas em um auditório da UEM, e por se tratar de uma oficina, havia um número limitado de vagas. Em 2020, ocorreu a quarta edição do evento, com os temas Odontologia Inclusiva: Comunicação básica em libras e Currículo Lattes, porém de forma remota, devido ao contexto de isolamento e distanciamento social que vivia-se na época. Dessa forma, as inscrições foram abertas 13 dias antecedentes ao evento por meio de uma plataforma online, na qual os participantes tiveram todas as informações do evento e receberam todos os materiais via e-mail. A oficina aconteceu online de maneira síncrona e os palestrantes se conectaram com os participantes por meio da plataforma Google Meet. Os links das reuniões foram enviados e disponibilizados aos inscritos uma hora antes do início das atividades via e-mail.

Ao final dos eventos, foram distribuídas avaliações a serem preenchidas pelos participantes, pedindo uma nota geral do evento e perguntando o que o participante achou da oficina, do tema, do conhecimento transmitido pelos ministrantes, da divulgação, da organização do evento, além de deixar espaço para críticas e ideias de melhorias para os próximos anos e também sugestões para os próximos temas, dessa forma, proporcionando feedbacks positivos e negativos do evento. Saber as necessidades e interesses dos participantes é importante, pois auxiliam na elaboração das futuras propostas didáticas e torna a atividade mais relevante para os estudantes, os quais terão maior interesse no assunto tratado (Martins et al., 2020).

\section{Resultados e Discussão}

A "I Oficina Acadêmica do Grupo PET Odontologia UEM", que ocorreu em 2017, reuniu 57 acadêmicos do curso de Odontologia em 2 dias de evento. Os temas abordados no primeiro dia da oficina "Confecção de resumo científico, painéis e apresentações no PowerPoint" são de suma importância para os acadêmicos de Odontologia, pois durante a graduação, os discentes apresentam diversos trabalhos científicos necessitam confeccionar resumos e apresentações adequadas, fundamentais para os acadêmicos obterem bons resultados. No segundo dia da oficina foi escolhido o tema "Currículo Lattes", devido à grande dificuldade dos alunos em preenchê-lo corretamente. O Currículo Lattes contempla o registro do que se produz cientificamente em âmbito nacional, auxiliando, dessa forma, na gestão da Ciência, Tecnologia e Inovação do país (Estácio, 2017). Durante a realização da oficina, os participantes também colocaram em prática o que estava sendo ensinado na oficina, e assim as dúvidas dos discentes foram sanadas pelas palestrantes. A avaliação desta atividade, por parte da comissão organizadora e dos inscritos, foi extremamente positiva e, por isso, os petianos decidiram continuar a realização da Oficina Acadêmica nos anos subsequentes.

Em 2018, aconteceu a "II Oficina Acadêmica do Grupo PET Odontologia UEM", que também reuniu 109 acadêmicos do curso de Odontologia em 2 dias de evento. No primeiro dia foi abordada a temática "Oratória", visto que grande parte dos alunos possuem grande dificuldade em expressar-se publicamente e saber comunicar-se corretamente. Ter uma boa oratória é fundamental na graduação, e esta tornou-se um fator chave para todas as áreas de atuação profissional, uma vez que enriquece 
a comunicação e as relações entre pessoas (Barcellos et al., 2006). Durante a realização da Oficina, os participantes entenderam a importância do saber se comunicar em público e houve discussões importantes a partir das dúvidas e apontamentos levantados. No segundo dia da oficina, foi abordado o tema "Excel". Ao decorrer da atividade, os palestrantes explicaram e realizaram atividades no Excel juntamente com os participantes, e conforme os participantes tinham dúvidas a respeito da dinâmica, os monitores davam o suporte necessário para solucionar o problema. O Excel é um excelente recurso para se trabalhar com questões de Estatística, visto que permite uma maior compreensão e interação com os conteúdos propostos (Silva et al., 2018), a realização desta atividade, de acordo com o feedback final, supriu as expectativas do grupo, já que foi amplamente elogiada pelos demais graduandos.

Em 2019, a "III Oficina Acadêmica do Grupo PET Odontologia UEM" obteve 48 acadêmicos do curso de Odontologia inscritos em 2 dias de evento. A oficina "Aprenda a administrar seu Tempo" foi elaborada devido à dificuldade dos alunos em planejarem sua rotina de forma simples e eficaz, dado que organizar as responsabilidades e conciliar o tempo com os planos futuros é um desafio enfrentado por muitos adultos atualmente (Oliveira et al., 2016). O gerenciamento de tempo corresponde a uma série de hábitos ou comportamentos que fornecem tempo efetivo, favorecendo a produtividade e reduzindo o estresse. Esses comportamentos podem ser adquiridos pela experiência de vida, treinamento ou prática (Claessens et al., 2007). Durante a oficina, os palestrantes mostraram o funcionamento de aplicativos, como: "Menthal"; "Forest"; "Steplock" e técnicas para motivar os participantes a colocarem em prática o que estava sendo ensinado na atividade. Os resultados foram extremamente positivos, de acordo com a avaliação final do evento, visto que vários acadêmicos relataram estarem organizando melhor sua rotina após a oficina. A oficina "Regras básicas da câmera fotográfica para o clínico e noções básicas de photoshop", também foi elaborada para auxiliar os alunos nestas temáticas. Na Odontologia, as fotografias realizadas antes, durante e após o tratamento, tornaram-se um documento valioso, permitindo um acompanhamento mais criterioso do caso, além da comparação dos benefícios proporcionados pelo tratamento proposto (Oliveira et al., 2011). No entanto, este tema não é abordado de modo prático em sala de aula. Assim, a temática escolhida despertou o interesse dos universitários, que participaram ativamente na execução dos exercícios sugeridos no momento da oficina, com a ajuda dos palestrantes. A avaliação final do evento por parte dos petianos e dos participantes foi positiva, já que os conteúdos foram transmitidos de modo simplificado e compreensível, facilitando a assimilação dos estudantes.

Em 2020, a "IV Oficina do Grupo PET Odontologia UEM" que ocorreu de forma remota devido a pandemia do COVID - 19, contou com a participação de 74 pessoas, inclusive de vários estados brasileiros, como: Paraná, Goiás, Pará, Rio Grande do Sul e o Distrito Federal. A Oficina de 2020, retomou um tema já abordado na edição de 2017, o Currículo Lattes, pois grande parte dos novos discentes não haviam participado da Oficina e, além disso, foi um dos temas mais solicitados pelos alunos da graduação, por meio das redes sociais do PET Odontologia UEM. O outro tema escolhido para o segundo dia de evento foi: "Odontologia Inclusiva: Comunicação básica em libras", sendo um grande diferencial da Oficina de 2020, uma vez que os palestrantes ensinaram aos participantes a se comunicarem com pessoas surdas, principalmente no contexto da Odontologia. Para ter sucesso no atendimento odontológico é preciso que a comunicação entre paciente e profissional seja bem estabelecida, visando compreender as queixas principais, o que o fez buscar um especialista, para compreender sua história médica, elaborar e explicar o plano de tratamento adequado para o caso em questão (Machado \& Silva, 2019). Exercer uma Odontologia inclusiva, pode proporcionar um atendimento individualizado e acolhedor ao paciente com necessidades especiais. A capacitação diferenciada dos futuros profissionais de Odontologia tem grande impacto no aspecto inclusivo, em particular no que diz respeito ao tratamento de pacientes com deficiência, dentre eles, os surdos (Machado \& Silva, 2019). E, apesar dessa edição ter sido realizada de modo totalmente online, houve uma grande interação com os participantes e palestrantes. Foi realizada a avaliação final do evento, por meio de um Formulário Google e os resultados foram extremamente positivos pelos inscritos e comissão organizadora. 
Os petianos exerceram seu efeito multiplicador junto aos colegas de graduação e pós-graduação ao colaborar na construção do conhecimento coletivo, além de adquirir experiência e habilidade em organizações de eventos. As quatro edições da oficina não tiveram intercorrências e foram cumpridas na sua integralidade. A equipe de organização se mostrou motivada a propor novas atividades nos próximos anos, pela tranquilidade com que os eventos transcorreram. Como dificuldades observadas na realização do evento, a comissão organizadora aponta a escolha de temas que sejam relevantes aos discentes e também a data do evento, para que ocorra a participação de grande parte da graduação. Especificamente no ano de 2020, a comissão organizadora teve dificuldade em realizar o evento de forma online, mantendo o mesmo padrão de interação entre os palestrantes e participantes. Os resultados satisfatórios das Oficinas Acadêmicas no curso de Odontologia da UEM já são notáveis, à medida que mais resumos são aceitos sem necessidade de correção e muitas apresentações de trabalhos são premiados em congressos. Ainda, os alunos relatam maior facilidade em elaborar planilhas no programa Excel, utilizar softwares de edição de fotos e organizar melhor suas rotinas.

As dúvidas dos estudantes sobre as temáticas foram sempre sanadas, além disso, a graduação sentiu-se satisfeita com as palestras e atividades. Portanto, o objetivo do grupo PET Odontologia foi alcançado tendo em vista que possibilitou a ampliação de experiências em formação acadêmica e cidadã, promovendo a formação ampla e de qualidade acadêmica, como previsto no MOB - Manual de Orientações Básicas do PET (Brasil, 2006).

\section{Considerações Finais}

A promoção de oficinas acadêmicas pelo grupo PET Odontologia UEM coopera com a melhoria do ensino da graduação, pois aborda temas que em geral não são apresentados em sala de aula. Ademais, são destinadas à socialização com os demais membros do curso, desenvolvendo habilidades e autonomia dos alunos. Quanto aos petianos, a responsabilidade de idealizar e colocar em prática atividades de ensino contribui na formação pessoal e profissional, estimula a didática, disciplina e integração da comissão organizadora.

Assim, pode-se concluir que é de suma importância a atuação dos grupos PET nos cursos de ensino superior, que por meio de suas atividades extracurriculares, ampliam o conhecimento acadêmico e o aperfeiçoamento da grade curricular como um todo.

\section{Referências}

Barcellos, C. A. P., Vieira, I. P., Carvalho, C. R. de B., \& Lopes, T. A. S. (2006). A oratória como um fortalecimento do marketing pessoal do profissional de administração. Janus, 3(4), 152-162.

Brasil. Ministério da Educação. Gabinete do Ministro. (2013, 25 de abril). Portaria n. ${ }^{\circ}$ 343, de 24 de Abril de 2013 . Diário Oficial da União, Brasília, DF. p. 24.

Brasil. Ministério da Educação. Gabinete do Ministro. (2010, 28 de julho). Portaria n. ${ }^{\circ}$ 976, de 27 de julho de 2010. Diário Oficial da União, Brasília, DF. p. 103 e 104.

Brasil. Ministério da Educação. Manual de orientações básicas. (2006). http://portal.mec.gov.br/pet/manual-de-orientacoes

Claessens, B. J. C., Eerde, van, W., Rutte, C. G., \& Roe, R. A. (2007). A review of the time management literature. Personnel Review, 36(2), 255-276. https://doi.org/10.1108/00483480710726136

Drebes, L. M., Ortigara, C., Artuzo, F., Jandrey, W., \& Silva, V. (2012). A dinâmica do Programa de Educação Tutorial (PET). Enciclopédia Biosfera, 8(15), 2334-2351.

Estácio, L. (2017). A importância do currículo lattes como ferramenta que representa a ciência, tecnologia e inovação no país. Revista ACB, 22 (2), 300-311.

Joaquim, F. F., \& Camargo, M. R. R. M. de. (2020). Revisão bibliográfica: oficinas. Educação em Revista, 36, e218538. https://doi.org/10.1590/01024698218538

Machado, L. K. P., \& Silva, F. A. P. (2019). Saúde e surdez: odontologia inclusiva. Encontro De Extensão, Docência E Iniciação Científica, 5(1). http://publicacoesacademicas.unicatolicaquixada.edu.br/index.php/eedic/article/view/3147 
Research, Society and Development, v. 10, n. 5, e48310514700, 2021

(CC BY 4.0) | ISSN 2525-3409 | DOI: http://dx.doi.org/10.33448/rsd-v10i5.14700

Manholer, A. E. de C., Shimada, A. F. B., Vasconcelos, C. F., Ricken, C. M., Morais, C. A. H. de, Kamikawa, D. S., Pagliosa, E. L., Henschel, F. A. N., Neme, G. S., Molina, L. M., Garcia, L. G. S., Ceron, L. C., Cardoso, L. G., Colombo Júnior, M., Fernandes, M. E., Andrade, M. P. de, Takahashi, R. Y., \& Petyk, W. S. (2021). Open your mind PET: Critical and citizen training promoted by students of the PET Dentistry group of Maringá State University. Research, Society and Development, 10(4), e17010414005. https://doi.org/10.33448/rsd-v10i4.14005

Marcondes, M. E. R. (2008). Proposições metodológicas para o ensino de Química: oficinas temáticas para a aprendizagem da ciência e o desenvolvimento da cidadania. Revista Em Extensão, 7(1), 67-77.

Martins, J. L. de C., Baptista, B. B., Oliveira, V. C. de, Martinez, A. D. W. V., Krindges, M., \& Braibante, M. E. F. (2020). Thematic workshop: the Chemistry in electronic devices. Research, Society and Development, 9(7), e997975271. https://doi.org/10.33448/rsd-v9i7.5271

Meneghel, S. N., Fonseca, E. de S. da, \& Silva, B. C. da. (2017). Oficina de bonecos: Experiências de um Programa de Educação pelo Trabalho (PET Saúde). In A. A. Ferla \& H. A. Pinto (Orgs.), Integração entre universidade e sistemas locais de saúde: experimentações e memórias da educação pelo trabalho (1st ed., pp. 312-334). Rede Unida.

Morel, L. L., Ferreira, N. B., Altmam, J. G., Silva, H. G., Chaves, E. T., \& Martos, J. (2020). Avaliação dos egressos do Pro grama de Educação Tutorial (PET) do curso de Odontologia da Universidade Federal de Pelotas. Revista da Abeno, 20(2), 119-130. https://doi.org/10.30979/rev.abeno.v20i2.1108

Nascimento, J. L. do, \& Feitosa, R. A. (2020). Active methodologies, focusing on teaching and learning processes. Research, Society and Development, 9(9), e622997551. https://doi.org/10.33448/rsd-v9i9.7551

Oliveira, C. T. de, Carlotto, R. C., Teixeira, M. A. P., \& Dias, A. C. G. (2016). Oficinas de Gestão do Tempo com Estudantes Universitários. Psicologia: Ciência e Profissão, 36(1), 224-233. https://doi.org/10.1590/1982-3703001482014

Oliveira, J. P., Polloni, D. G. O., \& Ignacio, F. (2011). A importância das fotografias posteriores no orçamento odontológico. Revista Dental Press de Estética. $8(1), 34-44$.

Paviani, N. M. S., \& Fontana, N. M. (2009). Oficinas pedagógicas: relato de uma experiência. Conjectura: filosofia e educação, 14(2), 77-88.

Pey, M. O. (1997). Oficina como modalidade educativa. Perspectiva, 15(27), 35-63.

Silva, M. dos S., Lima, R. S. N., \& Vieira, A. R. L. (2018). Excel como recurso didático nas aulas de estatística. Encontro Internacional de Formação de Professores e Fórum Permanente de Inovação Educacional, 11(1).

Souza, V. A. de. (2016). Oficinas pedagógicas como estratégia de ensino: Uma visão dos futuros professores de ciências naturais [Trabalho de conclusão de Curso, Licenciatura em Ciências Naturais, Universidade de Brasília. Planaltina, Distrito Federal, Brasil]. https://bdm.unb.br/handle/10483/14170 